Metastasiertes Prostatakarzinom

\section{Nutzen von Abirateron erneut belegt}

Die frühe Gabe von Abirateron in der Therapie von Patienten mit metastasiertem Prostatakarzinom verlängert die Überlebenszeiten signifikant, wie die Ergebnisse der LATITUDE-Studie zeigen. An der doppelblinden, randomisierten und placebokontrollierten Phase-II-Studie waren 1.199 Männer mit neu diagnostiziertem, metastatischem und hormonempfindlichem Prostatakrebs beteiligt. Sie erhielten randomisiert $1.000 \mathrm{mg} \mathrm{Abi-}$ rateron plus $5 \mathrm{mg}$ Prednison zusätzlich zur Androgendeprivationstherapie (ADT) oder ADT plus Placebo.

Das Gesamtüberleben nach einer medianen Nachbeobachtungszeit von 30,4 Monaten war in der Abirateron-Prednison-ADT-Gruppe signifikant länger als bei den Männern mit ADT und Placebo: Das mediane Überleben betrug bei letzteren 34,7 Monate, bei ersteren war der Median noch nicht erreicht $(\mathrm{p}<0,001)$. Die Mortalität in der Abirateron-Prednison-ADT-Gruppe war um $38 \%$ niedri- ger. Auch das radiologisch progressionsfreie Überleben war länger (33,0 vs. 14,8 Monate). Das Risiko für den Endpunkt Progression oder Tod reduzierte die Kombination Abirateron-PrednisonADT um $53 \%$.

In allen sekundären Endpunkten - darunter Schmerzprogression, nötige Folgetherapie, Beginn der Chemotherapie, PSA-Anstieg und Komplikationen am Skelett - schnitten die Patienten unter Abirateron besser ab.

$63 \%$ der Patienten in der AbirateronPrednison-ADT-Gruppe und $48 \%$ jener in der ADT-Placebo-Gruppe waren von unerwünschten Therapieeffekten vom Grad 3 oder 4 betroffen. Hypertonien und Hypokaliämien traten unter Abirateron-Prednison-ADT häufiger auf als unter ADT plus Placebo. Robert Bublak

Fizazi K et al. Abiraterone plus Prednisone in Metastatic, Castration-Sensitive Prostate Cancer: N Engl J Med. 2017;377(4):352-60.

\section{Niedriggradige Gliome}

Patienten mit niedriggradigen Gliomen profitieren auch langfristig mehr von einer Resektion als von einer zuwartenden Strategie. Einer norwegischen Studie zufolge leben Patienten nach einer frühzeitigen Operation signifikant länger als ohne diese Maßnahme. Für die Studie

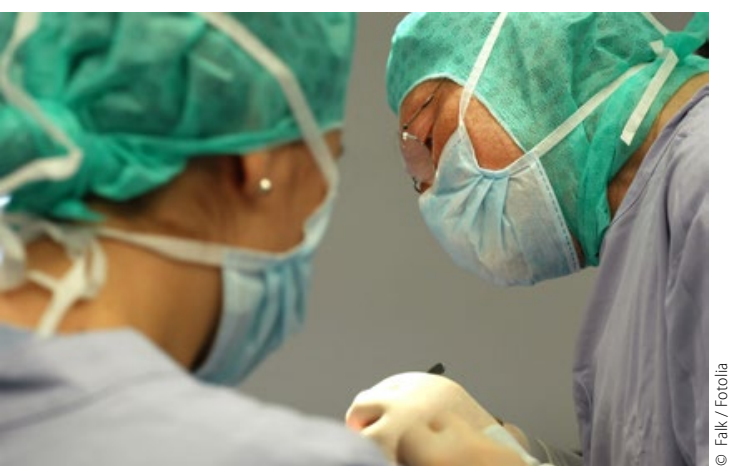

Bei Patienten mit niedriggradigen Gliomen lohnt sich eine Resektion. wurden die Befunde von 66 Patienten ausgewertet, bei denen eine abwartende Haltung (bis sich ein Tumorwachstum zeigte) Vorrang hatte, und von 87 Patienten mit bevorzugter frühzeitiger, u.a. 3-D-Sonografie-geführter Resektion. Zum einen ermittelten die Ärzte das Gesamtüberleben, zum anderen die krebsspezifischen molekularen Marker.

Die Diagnose der mindestens 18-jährigen Studienteilnehmer (Durchschnitt: 44 bzw. 45 Jahre) erfolgte zwischen 1998 und 2009. Das Follow-up endete Anfang Januar 2016. Bei $29 \%$ der 66 Patienten aus der Gruppe mit abwartender Strategie wurde eine Resektion vorgenommen, in der Resektionsgruppe bei $86 \%$. Von jeweils mehr als $90 \%$ (97 vs. $93 \%$ ) stand Gewebe für die Molekularanalyse zur Verfügung. Zwischen den Gruppen gab es keine unterschiedlichen Analyseergebnisse. Untersucht wurden der IDH(Isocitratdehydrogenase)-Status und

\section{kurz notiert}

Forschungsstandort Deutschland

Bessere Darmkrebs-Diagnostik +++ Sportmediziner der Johannes Gutenberg-Universität Mainz haben zusammen mit der Universitätsmedizin Mainz ein neues ultrasensitives Nachweisverfahren für DNA-Mutationen im Blut von Darmkrebspatienten entwickelt. Mit speziellen PCR-Techniken ist es gelungen, in einer geringen Blutmenge alle sieben K-RASMutationen sehr präzise zu quantifizieren. Damit ist es erstmals möglich, den Einfluss einer Operation auf die zirkulierende und die zellfreie DNA zu analysieren [Ehlert T et al. Sci Rep. 2017; 7(1):8876].

red.

Immunblockade bei $\mathrm{CLL}+++$

Wissenschaftler des Deutschen Krebsforschungszentrums untersuchten den Mechanismus, mit dem Krebszellen bei der CLL die körpereigene Immunabwehr umgehen. Dabei spielen Exosomen eine entscheidende Rolle. Die in Exosomen enthaltene nichtkodierende Y-RNA beeinflusst Monozyten derart, dass sie PD-L1 überexprimieren und so das Immunsystem drosseln. Diese Y-RNA wird von den Tolllike-Rezeptoren 7 und 8 erkannt, wodurch der Immuncheckpoint PD-L1 verstärkt exprimiert wird [Haderk F et al. Sci Immunol. 2017;2(13):pii eaah5509].

die 1p/19q-Kodeletion. Auch bei der Bestrahlung und der chemotherapeutischen Behandlung gab es keinen signifikanten Unterschied zwischen den beiden Studiengruppen.

Wie Asgeir Store Jakola und seine Kollegen berichten, lebten Patienten mit frühzeitiger Resektion signifikant länger als in der Vergleichsgruppe ( $\mathrm{p}=0,005)$. Die Gesamtüberlebenszeit lag der aktuellen Auswertung zufolge in der Gruppe mit abwartender Strategie bei 5,8 Jahren, in der Gruppe mit Operation dagegen bei 14,4 Jahren. Die Berücksichtigung molekularer Marker bei der Berechnung lieferte kein anderes Ergebnis.

Damit bestätigt die Auswertung der aktuellen Langzeitdaten das, was sich vor fünf Jahren angedeutet hatte. Die Ärzte weisen aber darauf hin, dass derzeit unklar ist, wie groß das erforderliche Ausmaß der Resektion sein muss, um einen Überlebensvorteil zu bieten.

Peter Leiner

Jakola AS et al. Surgical resection versus watchful waiting in low-grade gliomas. Ann Oncol. 2017; 28(8):1942-8. 